\title{
RISCOS QUÍMICOS A QUE ESTÃO SUBMETIDOS OS TRABALHADORES DE ENFERMAGEM: UMA REVISÃO DE LITERATURA ${ }^{1}$
}

\author{
Rosicler Xelegati ${ }^{2}$ \\ Maria Lúcia do Carmo Cruz Robazzi ${ }^{3}$
}

Xelegati R, Robazzi MLCC. Riscos químicos a que estão submetidos os trabalhadores de enfermagem: uma revisão de literatura. Rev Latino-am Enfermagem 2003 maio-junho; 11(3):350-6.

O estudo consiste em levantamento bibliográfico nas bases de dados LILACS, MEDLINE e ILO-CIS BULLETIN SAFETY AND HEALTH AT WORK sobre a temática riscos ocupacionais químicos, tendo como objetivo identificar, nas publicações encontradas, os principais agentes que podem favorecer o contato com esses riscos e provocar alterações de saúde nos trabalhadores de enfermagem. Foram encontrados 37 artigos, sendo apenas quatro publicados em periódicos nacionais que relatavam a detecção dos riscos químicos na manipulação de drogas citostáticas, na exposição a gases anestésicos, vapores de formaldeído/glutaraldeído e a gases esterilizantes, entre outros.

DESCRITORES: enfermagem, riscos ocupacionais

\section{CHEMICAL RISKS NURSING WORKERS ARE SUBJECT TO: A LITERATURE REVIEW}

This study consisted of a bibliographical survey in the databases LILACS, MEDLINE and ILO-CIS BULLETIN - SAFETY AND HEALTH AT WORK, with a view to identifying the main agents that can favor contact with these risks and cause health alterations in nursing workers. 37 articles were found, only four of which were published in national journals. Articles reported on the detection of chemical risks in the manipulation of cytostatic drugs; in the exposure to anesthetic gases, formaldehyde/ glutaraldehyde vapor and sterilizing gases, among others.

DESCRIPTORS: nursing, occupational risks

\section{RIESGOS QUIIMICOS A QUE ESTÁN SOMETIDOS LOS TRABAJADORES DE ENFERMERÍA: UNA REVISIÓN DE LITERATURA}

El estudio consiste en una revisión bibliográfica en las bases de datos LILACS, MEDLINE e ILO-CIS BULLETIN SAFETY AND HEALTH AT WORK y tiene como objetivo identificar los principales agentes que pueden favorecer el contacto con los riesgos químicos y provocar alteraciones de salud en los trabajadores de enfermería. Se encontraron 37 artículos, siendo solamente cuatro publicados en revistas nacionales. Los artículos abordaron la detección de riesgos químicos sobre la manipulación de drogas citostáticas; exposición a gases anestésicos, a vapores de formaldehido/glutaraldehido y a gases esterilizantes; etc.

DESCRIPTORES: enfermería; riesgos laborales

\footnotetext{
${ }^{1}$ Trabalho financiado pelo Programa Especial de Treinamento (PET/CAPES); ${ }^{2}$ Aluna do $4 \div$ semestre do Curso de Graduação, Bolsista do PET/ CAPES, e-mail: rxelegati@hotmail.com.br; ${ }^{3}$ Professor Titular, Orientadora do trabalho, e-mail: avrmlccr@eerp.usp.br. Escola de Enfermagem de Ribeirão Preto da Universidade de São Paulo, Centro Colaborador da OMS para o desenvolvimento da pesquisa em enfermagem
} 
INTRODUÇÃO

O hospital é o principal local de trabalho dos membros da equipe de enfermagem que, freqüentemente, permanecem a maior parte de sua vida produtiva dentro desse ambiente, muitas vezes em mais de um turno de trabalho, devido aos baixos salários ${ }^{(1-2)}$. Essa instituição, na qual se tenta salvar vidas e recuperar a saúde perdida das pessoas enfermas é a mesma que favorece o adoecer das pessoas que nela trabalham, porque, aparentemente, não há preocupação com a proteção, promoção $\mathrm{e}$ manutenção da saúde de seus empregados ${ }^{(2)}$.

A preocupação com a saúde do trabalhador de enfermagem fez-se presente desde 1700, através da publicação de Ramazzini, que questionou a contaminação das parteiras, possíveis precursoras dos profissionais de enfermagem, durante a realização de seu trabalho ${ }^{(3)} \mathrm{e}$ consolidou-se após o reconhecimento das ações de risco, através do uso dos equipamentos de proteção individual (EPI) e da relação dos agentes patogênicos com sua atividade profissional ${ }^{(4)}$.

No ambiente hospitalar há multiplicidade de riscos aos trabalhadores de enfermagem, sendo que, basicamente, parecem existir os: biológicos, físicos, químicos, psicossociais e ergonômicos. Os primeiros são os responsáveis por infeç̧ões agudas e crônicas, ocasionadas por vírus, fungos e bactérias. Os físicos são aqueles causados pelas radiações, vibrações, ruídos, temperatura ambiental, iluminação e eletricidade. Os riscos químicos são os gerados pelo manuseio de uma variedade grande de substâncias químicas e também pela administração de medicamentos que podem provocar desde simples alergias até importantes neoplasias. Os riscos psicossociais são desencadeados pelo contato com o sofrimento do paciente (estresse, fadiga mental, etc.). E os ergonômicos são gerados principalmente pela postura irregular dos profissionais de enfermagem em situações como movimentação de pacientes, flexões da coluna freqüentes, entre outros ${ }^{(1,5)}$.

O interesse pela temática e a tentativa de identificar o número de estudos existentes sobre os riscos ocupacionais para os trabalhadores de enfermagem despertaram para a realização de um levantamento bibliográfico geral, onde se constatou quantidade menor de estudos relacionados a riscos químicos em relação aos demais. Levando também em consideração a reduzida verbalização dos trabalhadores sobre os possíveis riscos de manipularem antibióticos de última geração, os problemas que podem ocorrer com o preparo de antineoplásicos, as dermatites que podem ser ocasionadas pelo uso prolongado de luvas protetoras e/ ou degermantes e outras substâncias químicas, utilizadas no ambiente hospitalar, justificaram a realização de um levantamento específico sobre a temática.

\section{OBJETIVO}

O objetivo desse estudo é identificar, através de artigos encontrados em bases de dados da área da saúde, as principais substâncias químicas às quais os trabalhadores de enfermagem estão expostos e os possíveis efeitos ocasionados em sua saúde.

\section{METODOLOGIA}

Para alcançar os objetivos propostos, realizou-se levantamento bibliográfico nas bases LILACS e MEDLINE, que são bases de dados gerais da Área da Saúde, através da via de acesso INTERNET, e o levantamento bibliográfico manual no periódico da Organização Internacional do Trabalho (OIT), chamado ILO-CIS BULLETIN - SAFETY AND HEALTH AT WORK, que é específica da Área de Saúde do Trabalhador.

A base MEDLINE foi analisada no período de 1979 a 2000, e a LILACS, no período de 1980 a 2000. Nessas bases de dados foram cruzadas as seguintes palavraschaves: enfermeira, trabalho de enfermagem, riscos ocupacionais químicos, saúde do trabalhador, "nurse", "working of nursing", "working chemical risks" e "occupational health".

Já o levantamento manual realizado no ILO-CIS BULLETIN-SAFETY AND HEALTH AT WORK foi efetuado no período de 1989 a 2000 em decorrência da não disponibilidade de volumes de anos anteriores. A análise dos estudos contidos nessa publicação realizou-se no tópico denominado "Nursing Personnel", encontrado no índice alfabético do final de cada volume do mencionado boletim.

Foram selecionados todos os artigos nacionais e internacionais que relacionavam a exposição a 
determinadas substâncias químicas como possíveis geradoras de riscos que pudessem ocasionar problemas à saúde dos trabalhadores de enfermagem, e tais estudos foram analisados através de ficha bibliográfica, mediante o resumo de todos os artigos. $\mathrm{Na}$ análise realizada, procurou-se investigar a quantidade de artigos encontrados em cada base bibliográfica e a síntese dessas publicações, com a identificação dos diferentes agentes químicos desencadeantes de patologias nos profissionais de enfermagem, segundo a distribuição absoluta e relativa dos diversos estudos encontrados.

\section{APRESENTAÇÃO E DISCUSSÃO DOS DADOS}

O levantamento bibliográfico realizado encontrou o total de 37 artigos sobre a temática abordada nas três bases de dados pesquisadas, sendo esses distribuídos na Tabela 1.

Tabela 1 - Distribuição de artigos localizados nas bases de dados ILO-CIS BULLETIN - SAFETY AND HEALTH AR WORK (1989-2000), LILACS (1980-2000) e MEDLINE (1979-2000), sobre os riscos ocupacionais químicos a que se submetem os trabalhadores de enfermagem

\begin{tabular}{ccccc}
\hline $\begin{array}{c}\text { BASEDEDADOS } \\
\text { ANODE PUBLCAÇÃO }\end{array}$ & $\begin{array}{c}\text { ILOCIS } \\
\text { BULIN }\end{array}$ & LLACS & MEDLNE & TOTAL \\
\hline 1979 & $*$ & $*$ & - & 0 \\
1980 & $*$ & - & - & 0 \\
1981 & $*$ & - & - & 0 \\
1982 & $*$ & - & 1 & 1 \\
1983 & $*$ & - & - & 0 \\
1984 & $*$ & - & - & 0 \\
1985 & $*$ & - & 1 & 1 \\
1986 & $*$ & - & - & 0 \\
1987 & $*$ & 2 & 1 & 3 \\
1988 & $*$ & - & - & 0 \\
1989 & 1 & - & - & 1 \\
1990 & 1 & - & 2 & 3 \\
1991 & 2 & - & 2 & 4 \\
1992 & 1 & - & - & 1 \\
1993 & 3 & - & 1 & 4 \\
1994 & 3 & - & - & 3 \\
1995 & 5 & 2 & 2 & 9 \\
1996 & 4 & - & - & 4 \\
1997 & 2 & - & - & 2 \\
1998 & - & - & - & 0 \\
1999 & - & - & - & 0 \\
2000 & 1 & - & - & 1 \\
\hline TOTAL & $\mathbf{2 3}$ & $\mathbf{4}$ & $\mathbf{1 0}$ & $\mathbf{3 7}$ \\
\hline & & & &
\end{tabular}

* Ano não pesquisado
Em relação ao levantamento realizado no ILO-CIS BULLETIN -SAFETY AND HEALTH AT WORK, no período de 1989 a 2000, foram encontrados apenas o total de 23 artigos, no período de doze anos em questão, sendo que não houve nenhuma publicação sobre a temática no ano de 1998 e 1999 e nenhuma menção a artigos em periódico nacional.

Encontrou-se o total de dez artigos na base de dados MEDLINE, no período de 1979 a 2000, sendo todas as publicações em periódicos distintos, incluindo-se um nacional. A quantidade de artigos sobre a temática é reduzida em comparação ao período de anos, e existe ausência de publicações nos períodos de 1979 a 1981, 1983 a 1984, 1986, 1988 a 1989, 1992, 1994 e de 1996 a 2000.

Na base de dados LILACS, no período de 1980 a 2000 , foram encontrados quatro artigos. Além do número de estudos ser reduzido em relação ao período, as publicações concentram-se nos anos de 1987 e 1995. Existe, portanto, uma lacuna em relação à temática riscos ocupacionais químicos dos trabalhadores de enfermagem nos períodos de 1980 a 1986, 1988 a 1994 e 1996 a 2000. Acresce-se a isso que a base de dados LILACS não é específica de temáticas envolvendo as questões Saúde e Trabalho, o que, de certa forma, auxilia na explicação do reduzido número de artigos encontrados, embora uma das revistas mencionadas (Rev. bras. saúde ocup.) seja específica sobre assuntos relacionados à Saúde do Trabalhador.

Outro fato interessante é que três dos quatro periódicos citados na base LILACS são nacionais. Portanto, apesar de, essa base de dados, estarem cadastrados periódicos estrangeiros, envolvendo várias regiões da América Latina e Caribe, há nela maior número de periódicos cadastrados brasileiros, o que exclui a possibilidade de se deduzir que os autores nacionais estão publicando mais sobre a Enfermagem e os Riscos Químicos de que os de outros países dessas regiões.

Quanto a síntese das publicações encontradas em todas as bases bibliográficas analisadas, pode-se agrupá-las, distribuindo-as conforme mostrado na Tabela 2. 
Tabela 2 - Número de publicações localizadas, segundo o tema abordado, mencionadas nas bases de dados ILOCIS BULLETIN - SAFETY AND HEALTH AR WORK (19892000), LILACS (1980-2000) e MEDLINE (1979-2000)

\begin{tabular}{|c|c|c|}
\hline SÍNTESE DAS PUBLICAÇŌES & $\begin{array}{c}\text { № DE } \\
\text { ARTIGOS }\end{array}$ & $\%$ \\
\hline Manipulação de drogas citostáticas & 12 & 32,5 \\
\hline Agentes químicos em geral & 8 & 21,6 \\
\hline Exposição a gases anestésicos & 5 & 13,5 \\
\hline $\begin{array}{l}\text { Exposição a vapores e gases } \\
\text { esterilizantes }\end{array}$ & 4 & 10,8 \\
\hline Manipulação do antibióticos cefalosporina & 2 & 5,4 \\
\hline Reações alérgicas a luvas protetoras & 1 & 2,7 \\
\hline Manipulação do propacetamol & 1 & 2,7 \\
\hline Exposição a vapores de cimento de osso & 1 & 2,7 \\
\hline $\begin{array}{l}\text { Terapia de aerosol com pentamidina e } \\
\text { ribavirina }\end{array}$ & 1 & 2,7 \\
\hline Manipulação de mercúrio & 1 & 2,7 \\
\hline $\begin{array}{l}\text { Transferência de produtos químicos para } \\
\text { o leite materno }\end{array}$ & 1 & 2,7 \\
\hline TOTAL & 37 & 100 \\
\hline
\end{tabular}

Sobre a manipulação de drogas citostáticas, podese observar que 12 artigos foram encontrados, relacionando a manipulação dessas drogas com a ocorrência de anormalidades cromossômicas, "troca de cromátides irmãs", câncer de pulmão em interação com o fumo, infertilidade e urticária.

A ocorrência de "troca de cromátides irmãs" são mais elevadas em enfermeiras expostas ao manuseio de drogas antineoplásicas do que aquelas não expostas. É importante ressaltar que a porcentagem de tal ocorrência tem um aumento insignificante em enfermeiras que, mesmo manipulando drogas citostáticas, trabalham em um ambiente seguro ${ }^{(6-7)}$. Porém, a freqüência de "troca de cromátides irmãs" em enfermeiras que trabalham sem equipamento de proteção individual é significativamente maior $^{(8)}$.

Outros autores também afirmam que não há aumento claro de danos cromossomais em enfermeiras expostas à manipulação de quantidades moderadas de drogas antineoplásicas em relação a um grupo não exposto, como o dos escriturários, mas reconhecem que as aberrações cromossômicas são significativamente mais elevadas no grupo das enfermeiras, em relação aos escriturários ${ }^{(9)}$.

Outro fator importante a ser considerado é a interação do fumo com a manipulação de drogas citostáticas, que pode causar desde a "troca de cromátides irmãs"(10) até câncer de pulmão ${ }^{(11-12)}$.

Os relatos sobre infertilidade também foram encontrados entre enfermeiras que manipulam drogas antineoplásicas $^{(13)}$.

Uma enfermeira apresentou urticária no rosto, tórax, membros superiores e pés 30 minutos após administrar uma solução de infusão de cisplatina, um tipo de quimioterápico ${ }^{(14)}$

Os demais artigos encontrados, que retratavam o manuseio de citostáticos, relatavam os perigos de saúde específicos das enfermeiras ao manipularem antineoplásicos $^{(15)}$ e faziam recomendações para preparação, administração e disposição desse tipo de drogas, visto que a preparação dessas sem proteção pode conduzir a mutações ${ }^{(16)}$. Há autores solicitando revisão das normas para manipulação das drogas antineoplásicas $^{(17)}$, tendo em vista a importância do trabalhador estar devidamente protegido para entrar em contato com essas substâncias.

Oito dos artigos analisados explicavam os principais agentes químicos que causam patologias de origem ocupacional, na área hospitalar. Os principais agentes mencionados foram as drogas antineoplásicas, os agentes esterilizantes, os gases anestésicos, entre outros e esses podem estar causando desde dermatites e problemas reprodutivos, até neoplasias.

Como já referido, os hospitais apresentam uma variedade de riscos, dentre os quais os riscos químicos parecem estar relacionados mais intensamente com as drogas antineoplásicas, os gases anestésicos, os agentes esterilizantes e os irritantes de pele ${ }^{(18-21)}$. Esses agentes químicos podem provocar nos trabalhadores intoxicações agudas e estados crônicos e pré-patológicos de várias doenças ocupacionais ${ }^{(22)}$.

A exposição a gases anestésicos, antineoplásicos e esterilizantes é potencialmente prejudicial aos processos reprodutivos (abortos espontâneos, defeitos congênitos, infertilidade) e deve-se minimizar essa exposição principalmente em trabalhadoras grávidas ou que planejam engravidar $^{(23)}$.

A descendência de trabalhadores expostos ocupacionalmente a gases anestésicos, gases esterilizantes, mercúrio e radiações $X$ corre o risco significativo de apresentar problemas como anencefalia, espinha bífida, defeitos no sistema urinário e genital ${ }^{(24)}$.

Há programas de controle biológico da exposição dos trabalhadores aos riscos químicos, porém, se esses programas não forem realizados de maneira criteriosa, acabam submetendo os trabalhadores a experimentações $^{(25)}$. 
Cinco foram as publicações sobre a exposição a gases anestésicos encontradas e que podem estar influenciando a ocorrência de malformações congênitas, abortos espontâneos, "performance" diminuída da capacidade de trabalho ${ }^{(26)}$ e diminuição da produção de DNA entre trabalhadores de enfermagem.

A freqüência de ocorrência de abortos espontâneos é significativamente mais elevada em enfermeiras que trabalham em salas de operação durante a gravidez ${ }^{(27)}$. Essa constatação da influência negativa do óxido nitroso, durante a gravidez, foi pesquisada e confirmada, até em relação às parteiras nascidas em $1940^{(28)}$.

A poluição do ar das salas de operação pelos gases anestésicos (N2O) produz efeitos adversos nos trabalhadores, porque o óxido nitroso oxida a vitamina B12 e assim diminui a produção de DNA por inativação da síntese de metionina ${ }^{(29)}$. O problema da poluição de gases anestésicos existe e preocupa até governos, como o Reino Unido, que publicou relatório afirmando que a problemática de poluição para os trabalhadores da área de saúde ainda não está resolvida ${ }^{(30)}$.

Outro risco ocupacional químico encontrado foi o proporcionado pela exposição a vapores de formaldeído e glutaraldeído e a gases esterilizantes (óxido de etileno). Os quatro artigos encontrados discutiam o tema, destacando o desenvolvimento de reações alérgicas (dermatites, renites, entre outras) até a ocorrência de abortos espontâneos.

O aparecimento de reações alérgicas, causadas pelo uso do glutaraldeído, já foi confirmada ${ }^{(31)}$. Um dos estudos relatou o caso de enfermeiras e médicos de uma unidade de endoscopia que passaram a apresentar manifestações clínicas como lacrimejamento dos olhos, rinites, dermatites, dificuldades respiratórias, náuseas e dor de cabeça após a exposição ao glutaraldeído $2 \%{ }^{\left({ }^{(32}\right)}$. O contato da pele com desinfectantes como os formaldeídos também pode causar alergias ${ }^{(33)}$.

A freqüência aumentada da ocorrência de abortos espontâneos entre o pessoal que trabalha em procedimentos de esterilização, principalmente durante a gravidez, apresenta correlação com a exposição a óxido de etileno, mas não com a utilização de glutaraldeído ou formaldeído ${ }^{(34)}$.

Dois artigos relataram o risco da manipulação de antibióticos como as cefalosporinas, entre eles um que relatou o caso de uma enfermeira que apresentou sarna/ coceira eritematosa em vários trechos da pele (rosto, braços e mãos) após a preparação de soluções de cefalosporinas. Testes indicaram que a dermatite de contato alérgica ocupacional deve-se à exposição à $1^{\underline{a}}, 2^{\underline{a}}$ e $3^{a}$ gerações de cefalosporinas ${ }^{(35)}$. As cefalosporinas podem causar alergias e rinites alérgicas ${ }^{(36)}$.

Em relação ao manuseio constante de luvas protetoras, um artigo relatou a ocorrência de dermatite de contato, asma e rinites ${ }^{(37)}$.

Casos de dermatite de contato ocupacional foram identificados em enfermeiras ao prepararem injeções do analgésico propacetamol. As mesmas enfermeiras não apresentaram alergia com um outro analgésico, como o paracetamol. Os trabalhadores da área de saúde estão aconselhados a usar luvas de proteção quando manipularem propacetamol ${ }^{(38)}$.

Uma das publicações apresentou o caso de uma enfermeira que desenvolveu úlcera na córnea repetidamente enquanto misturava cimento de osso, provavelmente devido aos vapores provenientes do mesmo ${ }^{(39)}$.

A toxicidade da terapia de aerosol com antiprotozoário (pentamidina) e antiviral (ribavirina) também foi constatada em um dos artigos. As pequenas partículas dessas drogas são de difícil controle e prejudicam a saúde dos trabalhadores expostos ${ }^{(40)}$.

A manipulação do metal mercúrio provocou dermatite de contato alérgica em uma enfermeira dental que manipulou o material sem luvas protetoras ${ }^{(41)}$.

Outro artigo informava os perigos da transferência de produtos químicos, que as mães entram em contato no ambiente de trabalho, para o leite materno, possibilitando a ingestão desses produtos pelos bebês ${ }^{(42)}$.

\section{CONCLUSÕES FINAIS}

As publicações referentes à proteção dos trabalhadores de enfermagem, principalmente em relação aos riscos químicos que esses enfrentam, ainda é muito reduzida e o que existe publicado concentra-se em periódicos internacionais.

Os administradores de hospitais e os trabalhadores da área de saúde parecem ainda não conseguirem enxergar que a prevenção dos riscos ocupacionais gera vantagens em todos os níveis, já que se constituem em lucro para os hospitais, saúde para seus trabalhadores e publicações notáveis para os pesquisadores. 
Mais pesquisas sobre essa temática poderiam

ser realizadas, com o intuito de aumentar o conhecimento existente sobre a mesma, facilitando-se a tomada de
1. Bulhões I. Riscos do trabalho de enfermagem. $2^{\circ}$ ed. Rio de Janeiro (RJ): Editora Folha Carioca; 1994.

2. Takeda E. Tuberculose: um estudo de sua situação entre pacientes internados e equipe de enfermagem de um hospital escola de Ribeirão Preto, São Paulo. [dissertação]. Ribeirão Preto (SP): Escola de Enfermagem de Ribeirão Preto/ USP; 1996.

3. Mendes R. Patologia do trabalho. Rio de Janeiro (RJ): Atheneu; 1995.

4. Steagall-Gomes DL. Estudo dos riscos à saúde das pessoas que trabalham em enfermagem hospitalar. Rev Paul Hosp 1986; 34(4-6):85-93.

5. Marziale MHP. Condições ergonômicas da situação do pessoal de enfermagem em uma unidade de internação hospitalar. [tese]. Ribeirão Preto (SP): Escola de Enfermagem de Ribeirão Preto/USP; 1995.

6. Milkovic-Kraus S, Horvat D. Chromosomal abnormalities among nurses occupationally exposed to antineoplastic drugs. Am J Ind Med 1991; 19(6):771-4.

7. Brumen V, Horvat D, Trosic I. Potential genotoxic risk related to simultaneous exposure to radionuclides and cytostatics. Am J Ind Med 1995; 27(6):871-6.

8. Brumen $V$, Horvat $D$. Work enironment influence on cytostatics-induced genotoxicity in oncologic nurses. Am J Ind Med 1996; 30(1):67-71.

9. Sarto F, Trevisan A, Tomanin R, Canova A, Fiorentino M. Chromosomal aberrations, sister chromatid exchanges, and urinary thioethers in nurses handling antineoplastic drugs. Am J Ind Med 1990; 18(6):689-95.

10. Górecka D, Górski T. The influence of cigarette smoking on sister chromatid exchange frequencies in peripherad lymphocytes among nurses handling cytostatic drugs. Polish J Occup Med Environ Health 1993; 6(2):143-8.

11. Lagorio S, Forastiere F, Rapiti E, Di Pietro A, Costa G. Economic and occupational activities at increased risk of mortality from lung tumours in Turin (1981-82) and Italy (198182). Med Lav 1995; 86(4):309-24.

12. Wunsch V Filho. Riscos ocupacionais e câncer de pulmão. J Pneumol 1995; 21(1):34-42.

13. Valanis B. Occupational exposure to antineoplastic agents and self-reported infertility among nurses and pharmacists. J Occup Environ Med 1997; 39(6):574-80.

14. Schena D, Barba A, Costa G. Occupational contact urticaria due to cisplatin. Contact Dermatitis 1996; 34(3):220-1.

15. Hewit JB, Misner ST, Levin PF. Health hazards of nursing: identifying workplace hazards and reducing risks. AWHONNS Clin Issues Perinat Womens Health Nurses 1993; 4(2):3207 .

16. Kaijser GP, Underberg WJ, Beijnen JH. The risks of handling cytotoxic drugs II. Recommendations for working with cytotoxic drugs. Pharm Weeke Sci 1990; 12(6):228-35.

17. Mcdiarmid MA, Gurlcy HT, Arrington D. Pharmaceuticals as hospital hazards: managing the risks (see comments). J Occup Med 1991; 33(2):155-8.

18. Guidotti TL. Occupational health for hospital workers. Am Fam Physician 1987; 35(2):137-42.

19. Harrington JM. The health of health care workers. J R Colle Physicians Lond 1990; 24(3):189-95. consciência dos próprios trabalhadores de enfermagem, as principais vítimas dos problemas ocupacionais relacionados aos riscos químicos.

20. Tan CC. Occupational health problems among nurses. Scand J Work, Environ Health 1991; 17(4):221-30.

21. Barbosa A. Hospitais: fontes de saúde ou de riscos? Rev Saúde Dist Fed 1995; 6(1/2):32-6.

22. Durão A. La educación de los trabajadores y su participación en los programas de salud ocupacional. Educ Méd Salud 1987; 21(2):117-33.

23. Ahlborg G Júnior, Hemminki K. Reproductive effects of chemical exposure in health professions. J Occup Environ Med 1995; 37(8):957-61.

24. Matte TD, Mulinare J, Erickson JD. Case-control study of congenital defects and parenteral employment in health care. Am J Ind Med 1993; 24(1):11-23.

25. Buschinelli JTP. Utilização de indicadores biológicos em controle médico de trabalhadores. Rev Bras Saúde Ocup 1987; 15(58):33-9.

26. Lucchini R, Toffoletto F, Camerino D, Fazioli R, Guittori S, Gigliori R, et al. Neurobehavioral functions in operating theatre personel exposed to anesthetic gases. Med Lav 1995; 86(1):27-33.

27. Saurel-Cubizolles MJ, Hays M, Estryn-Behar M. Work in operating rooms and pregnancy outcome nurses. Int Arch Occup Environ Health 1994; 66(4):235-41.

28. Ahlborg G, Axelsson G, Bodin L. Shift work, nitrous oxide exposure and subfertility among Swedish midwives. Int $\mathrm{J}$ Epidemiol 1996; 25(4):783-90.

29. Hoerauf K, Koller C, Wiesner G, Taeger K, Hobbhalm J. Personnel's of operational room nitrous oxide exhibition in intubation anesthesia. Gesundheitswesen 1995; 57(2):92-6. 30. Halsey MJ. Occupational health and pollution from anaesthetics: a report of a seminar. Anaesthesia 1991; 46(6):486-8.

31. Geier J. Glutaraldehyde - occupations connected with na allergen. Derm Beruf Umwelt 1995; 43(1): 30-1.

32. Jachuck SJ, Bound CL. Occupational hazard in hospital staff exposed to 2 per cent glutaraldehyde in on endoscopy unit. J Soc Occup Med 1989; 39(2):69-71.

33. Czeschinski P. Tortured hands - occupational skin diseases in the hospital. Krankenhaus Technik 1994; 20(8):32-5.

34. Hemminki K, Mutanen P, Saloniemi I, Niemi ML, Vainio H. Spontaneous abortions in hospital staff engaged in sterilising instruments with chemical agents. Br Med J (Clin Res Ed) 1982; 285(6353):1461-3.

35. Filipe P, Almeida RSLS, Rodrigo FG. Occupational allergic contact dermatitis from cephalosporins. Contact Dermatitis 1996; 34(3):226.

36. Foti C, Bonamonte D, Trenti R, Venã GA, Angelini G. Occupational contact allergy to cephalosporins. Contact Dermatitis 1997; 36(2):104-5.

37. Zotti R, Muran A, Negro C. Follw-up of symptons in a group of health care workers sensitised to latexFollow-up of allergic symptoms among nurses sensitised to látex. Med Lav 2000; 91(1): 53-60.

38. Barbaud A, Trechot P, Bertrand A, Schmutz JL. Occupational allergy to propacetamol. Lancet 1995; 346(8979):902.

39. Nissen JN, Corydon L. Corneal ulcer after exposure to vapours from bone cement (methyl methacrylati and hydroquinone). Int Arch Occup Environ Health 1985; 56(2):1615. 
40. Roux EB. Riks among health care personnel using ribavirin and pentamidine aerosol therapy - DMT59 TC50, DMTDocuments pour le médecin du travail 1994; 59:259-65.

41. Kanerva L, Komulainen M, Estlander T, Jolanki R. Occupational allergic contact dermatitis from mercury. Contact Dermatitis 1993; 28(1):26-8.

42. Giroux D, Lapointe G, Baril M. Toxicological index and the presence in the workplace of chemical hazards for workers who breast-feed infantis. Am Ind Hyg Assoc J 1992; 53(7):471-

4. 